\title{
Ultraviolet-induced birefringence in hydrogen-loaded optical fiber
}

\author{
Canning, John; Deyerl, Hans-Jürgen; Sørensen, Henrik Rokkjær; Kristensen, Martin
}

Published in:

Journal of Applied Physics

Link to article, DOI:

$10.1063 / 1.1856215$

Publication date:

2005

Document Version

Publisher's PDF, also known as Version of record

Link back to DTU Orbit

Citation (APA):

Canning, J., Deyerl, H-J., Sørensen, H. R., \& Kristensen, M. (2005). Ultraviolet-induced birefringence in hydrogen-loaded optical fiber. Journal of Applied Physics, 97(5), 053104. https://doi.org/10.1063/1.1856215

\section{General rights}

Copyright and moral rights for the publications made accessible in the public portal are retained by the authors and/or other copyright owners and it is a condition of accessing publications that users recognise and abide by the legal requirements associated with these rights.

- Users may download and print one copy of any publication from the public portal for the purpose of private study or research.

- You may not further distribute the material or use it for any profit-making activity or commercial gain

- You may freely distribute the URL identifying the publication in the public portal

If you believe that this document breaches copyright please contact us providing details, and we will remove access to the work immediately and investigate your claim 


\title{
Ultraviolet-induced birefringence in hydrogen-loaded optical fiber
}

\author{
J. Canning, ${ }^{\text {a) }}$ H. J. Deyerl, ${ }^{\text {b) }}$ H. R. Sørensen, and M. Kristensen ${ }^{\text {c) }}$ \\ Research Center for Communications, Optics and Materials (COM), \\ Technical University of Denmark, Ørsteds Plads, Building 345 V, DK-2800 Kongens Lyngby, \\ Denmark
}

(Received 12 July 2004; accepted 15 December 2004; published online 11 February 2005)

\begin{abstract}
A precision phase-shifting approach to fabricate various phase-shifted gratings using different combinations of polarized ultraviolet (UV) light is demonstrated. In doing so, the difference between $s$ - and $p$-polarized light reported by others is confirmed. However, we reveal added complexity for the role of hydrogen and deuterium in the UV-induced process. Previous arguments for the origins are systematically ruled out by reviewing existing literature. We note that the birefringence is made up of at least two components with different thermal stabilities, one consistent simply with molecular hydrogen being present in the system. Overall the birefringence, by deduction, is associated with anisotropy in hydrogen reactions within the fiber. As a result they lead, through known mechanisms of dilation in glass, to anisotropic stress relaxation that can be annealed out, with or without hydrogen remaining, at low temperatures close to $125^{\circ} \mathrm{C}$. (C) 2005 American Institute of Physics. [DOI: 10.1063/1.1856215]
\end{abstract}

\section{INTRODUCTION}

Molecular hydrogen is critical to the production of fiber Bragg gratings with low material loss and high-induced index changes. Its isotope, deuterium, is even more significant, albeit also more expensive and hence often not used except in critical applications, since it shifts the absorption overtones of induced hydrogen species away from the telecommunications window. In both cases the most common method of introducing hydrogen into the system is by highpressure (commercial systems are capable of going up to 2000-atm pressure) diffusion of the molecules into the glass ${ }^{1}$ which up until $\sim 400 \mathrm{~atm}$ follows a near-ideal gas behavior. Beyond this, interactions with itself in the gas phase lead to increasing liquidlike behavior resulting in a complex diffusion profile. ${ }^{2}$

Ultraviolet (UV)-induced birefringence is a result of UVinduced effective index changes for the two polarization eigenstates that are not equal-the difference leads to a modal birefringence that usually exceeds the intrinsic fiber birefringence. Despite its obvious significance the exact role of hydrogen and its isotope in index change is unclear: both hydride and hydroxyl species are thought to be critical, with the former mainly responsible for core index change leading to increased densification of the glass compared to the irradiated pristine state. From hypersensitization experiments the time scales involved for the formation of these species indicate that $\mathrm{OH}$ is a final product and that a complex catalytic pathway between the two exists. ${ }^{3-5}$ There is evidence, on the other hand, that $\mathrm{OH}$ correlates with an initial negative index

\footnotetext{
${ }^{a}$ Present address: Optical Fibre Technology Centre, ATP, University of Sydney, Sydney, NSW 1430, Australia; electronic mail: j.canning@oftc.usyd.edu.au

${ }^{b}$ Present address: Institut für Physik, Technische Universität Chemnitz, D-09107 Chemnitz, Germany.

${ }^{c}$ Present address: Institute of Physics and Astronomy, Aarhus University, Ny Munkegade, Building 520, DK-8000 Århus C, Denmark.
}

change in fully loaded phosphosilicate optical fibers which is subsequently dominated by a positive index change thought to arise from a photorefractive effect. ${ }^{6}$ A recent approach to analyzing the characteristic curves of irradiated materials ${ }^{7}$ indicates that the linear growth in positive index change, after the initial negative contribution to dilation is swamped, with $\mathrm{OH}$ formation as a function of fluence is indicative of a photorefractive process. Any other process involves local or collective glass structural relaxations that are characterized by exponential relations. Given the generic nature of dilation associated with $\mathrm{OH}$ (or just the presence of molecular hydrogen), the first part of the characteristic curve for hydrogenloaded silicate-based fiber generally, can be characterized this way. This is despite the differences between phosphosilicate and germanosilicate chemistries, such as the transient index change in unhydrogenated phosphosilicate optical fiber, ${ }^{8}$ the deviation from a correlation with pure mechanical diffusion of $\mathrm{H}_{2}$ into phosphosilicate fiber, ${ }^{8}$ and the difference in magnitude and correlations of photodarkening and index change between these fibers. ${ }^{9}$

More direct experimental evidence of dilation in bulk nonphosphosilicate glass irradiated with low-energy exciplex emission, in the one-photon excitation regime, from an ArF laser has been reported. ${ }^{10}$ At low fluencies rarefaction, or dilation, is observed and the bulk induced birefringence follows the laser polarization though the authors were not entirely convinced of the connection. At higher fluencies, densification is observed and a tangential vector in the bulk birefringence associated with stress around the densified region was mapped. Smith et al. ${ }^{11}$ reported similar results for hydrogen-loaded glass irradiated with a 248-nm light and inferred that $\mathrm{OH}$ formation leads to this dilation along with a positive photorefractive index change. They suggest that water impurities in the previous work are involved. In addition, hydrogen penetration into a material is generally known to cause dilation of the lattice, ${ }^{12,13}$ a process not considered in glass fiber systems until now. Hence there appears to be 
some consistency between bulk glass and optical fiber measurements, although the latter system is far more complex, involving additional viscous and thermal stresses generated in a drawn bimaterial system. The inherent asymmetry may itself play a number of roles in enhancing the anisotropic effects of stress and preferential alignment of structure radially, and therefore the spatial distribution of hydrogen reaction species that may form, including any dipole orientation, within loaded systems.

In this paper, we present a comprehensive study of the UV-induced birefringence in hydrogen-loaded optical fibers. We discuss the results and their relation to any anisotropy in the induced index changes. From this, we conclude that molecular hydrogen creates stress relief of the core/cladding viscous and thermal stresses probably through partial dilation by the complex interactions of hydrogen with the glass. Initially, a simple physical swelling of the system occurs, analogous to the observation of visible swelling of polymer or Kevlar-based $\mathrm{O}$ rings used in some hydrogen-loading systems, for example, which mostly recover once out diffusion takes place. Stress anisotropy occurs when the hydrogen interactions are anisotropic as a result of the anisotropic absorption of UV light across the core, leading to the anisotropic depletion of molecular hydrogen and a small amount of index anisotropy.

The following sections are thus divided: in Sec. II we overview intrinsic optical fiber birefringence and existing work on UV-induced birefringence and critically examine some of the previous theories and ideas. Section III describes the method we use to study the induced birefringence. The phase-shifting approach to grating writing we have recently reported ${ }^{14}$ is described in the context of the polarization control grating writing method ${ }^{15,16}$ since we can use this method to produce unambiguously various phase-shifted gratings using all $s$-polarized light, $p$-polarized light, and a phaseshifted grating with half its length written by $p$-polarized light and the other half with $s$-polarized light. Note that we follow the convention introduced by Erdogan and Mizrahi ${ }^{17}$ and others that $s$ is the writing beam polarization orthogonal to the fiber axis and $p$ is parallel. Some discussion of the methods employed and their limitation is necessary for reasons to be given. The results are consistent with past measurements on bulk exposures by conventional means. Section IV presents the results in measuring the UV-induced birefringence and compares and discusses the differences between the various grating structures as well as with previous bulk measurements. Section V presents annealing and outdiffusion results on the UV-induced birefringence. Section VI moves on to discussing all these results and comparing previous work to see whether some of the critique in Sec. II is valid. We also comment on their implications in existing and future device applications both in terms of fiber properties and performance. Finally, Sec. VII states our conclusions on the role of hydrogen both in UV-induced birefringence and, more generally, in photosensitivity.

\section{BIREFRINGENCE IN OPTICAL FIBERS}

\section{A. Intrinsic fiber birefringence}

While we do not wish to go into any depth concerning the nature and properties of intrinsic fiber birefringence, it is evident that mechanical properties of the glass may change through hydrogen dissolution and/or irradiation, particularly since weak hydrogen bonding within the network occurs. It is also evident that as a result of a radial stress at the core/ cladding interface preferential orientation or defect localization may occur at this interface. The role of hydrogen bonding is unclear but is present-one of us has found that loading a fiber with a noninteracting species such as inert $\mathrm{He}$ leads to a decrease in photosensitivity, ${ }^{18}$ presumably through a reduction in the degrees of freedom for structural relaxation to occur favoring a greater rate of recombination. The existing anisotropy may therefore, in the presence of an interactive species such as $\mathrm{H}_{2}$, lead to an enhanced anisotropic response to UV irradiation along the fiber birefringence axes. Evidence supporting this notion can be found in experiments on high birefringence fibers where selective excitation of the fast and slow axes using $200-\mathrm{MeV}$ protons led to significantly different transient absorption rates at $1300 \mathrm{~nm}$ for each axis. ${ }^{19}$ Wong et al. ${ }^{20}$ also found that UV irradiation of a high birefringence optical fiber led to a reduction in the birefringence, which was interpreted by one of the authors as a stress relief mechanism that underpinned photosensitivity generally. ${ }^{21}$ However, since the experiment only measured birefringence it produced no evidence that stresses had decreased and the subsequent application of this idea to grating photosensitivity was found to be incorrect. ${ }^{22}$ Instead, the evidence suggested that anisotropic stress relief has occurred, i.e., a principle of a system equilibrating it stresses when the energy to do so is present. This principle was later invoked to explain the enhanced stability of type-IIa fiber gratings. ${ }^{23}$ Both the two examples thus mentioned contemplate the contribution of existing anistropy in very high birefringence optical fibers where the birefringence is determined by external parameters. In the second case, where the imparted energy is lower, the changes observed are milder and tend to satisfy equilibration of the external stress fields giving rise to elastic changes in the core birefringence. In nonbirefringent fibers the intrinsic birefringence is effectively a form birefringence since to remove the frozen in elastic strains annealing close to the glass transition is required. ${ }^{17}$ Therefore, the role of this intrinsic anisotropy, together with the presence of radial stresses, in enhancing UV-induced birefringence is potentially important but its details have not been discussed.

\section{B. UV-induced birefringence}

The origin of induced birefringence has been ascribed initially to two mechanisms: (1) induced form birefringence arising from a finite attenuation of UV light across the core through defect absorption and (2) preferential dipole excitation as a result of the laser writing polarization. ${ }^{24,25}$ Some evidence supporting both cases has been put forth, but in all cases the interpretations are inconclusive and may be coincidental within the limits of the conducted experiments. For example, UV etching showed that irradiated nonhydroge- 
nated fibers from one side reveal an anisotropy ${ }^{26}$ which can be reduced by then irradiating from the other side. This purportedly leads to a corresponding reduction in measured birefringence. However, while logical and certainly expected, the interpretation of the etching results has to be done with great care since etching is known to be highly chemically sensitive while being comparatively insensitive to structural change and stress fields ${ }^{27}$ such as those involved with nonhydrogenated and hypersensitized optical fibers. ${ }^{4,5}$ Hence, the large anisotropic etching depths observed are in agreement with an anisotropic defect generation or reaction apparently located at the core/cladding interface.$^{28}$ It is especially important to note, but not commented on, that hydrogen loading has been employed in these experiments. Induced birefringence observed in hydrogen-loaded optical fibers can reach values as high as $5 \times 10^{-4}$ over very long exposures. The UV-induced birefringence in unloaded optical fibers, on the other hand, is substantially smaller and often commensurate in magnitude with the intrinsic birefringence. Hence, it is not clear how the results translate to an anisotropic index change.

In hydrogen-loaded optical fibers, therefore, a number of difficulties exist with a simple index anisotropy of the type alluded to in the previous etching reports: First, index profiling across the core of heavily irradiated and very high pressure loading $(>700 \mathrm{~atm})$ shows little evidence of highly localized asymmetry $(<0.5 \mu \mathrm{m})$ in the index profile, ${ }^{29}$ which indicates that the UV index change in loaded optical fibers remains relatively uniform. However, in contrast it is worth pointing out that even with the low spatial resolution $(\sim 1 \mu \mathrm{m})$ of these types of optical measurements some asymmetry over several microns across the entire core is present. Another point is the role of stress and asymmetry in its contribution to index change and in dipole alignment. On their own, therefore, these results are so far inconclusive and have not been quantified in the literature. Second, the etching results were reported before it was recognized that corecladding defects are not the same as core defects and changes there do not necessarily contribute to the index change-for example, core/cladding interface defects luminesce in the visible much more strongly than core defects prior to illumination but are swamped by core defect-induced luminescence afterwards. ${ }^{30}$ Further, photodarkening in the core itself has been shown to have no obvious relation to the induced index change ${ }^{9}$ and hence a chemical or photochromic change alone is not evidence of significant index change in fibers. Third, the standard measurement of induced birefringence is actually a measure of waveguide modal birefringence-that is the difference in propagation constants between the two polarization states. Since intrinsic birefringence is generally low $\left(\sim 1 \times 10^{-6}\right)$ the differential fraction of light between eigenstates seeing this region is small. Therefore, it is not clear that this surprisingly narrow crescent $(<0.5 \mu \mathrm{m})$ observed right at the core/cladding boundary is solely responsible for the relatively large modal birefringence of UVinduced birefringence in hydrogen-loaded standard photosensitive fibers (typically $\sim 10^{-5}-10^{-4}$ at high fluencies). Lastly, some doubt on the specific interpretation based on using etching exists when hydrogen is involved since etching is predominantly chemical in nature, with changes arising from structural density changes being comparatively small. Hydrogen enhances the chemical selectivity of etching and the localization into a crescent at the core/cladding interface turns out to be of considerable interest for another reason. For example, in the case of hydrogen-loaded optical fibers chemical selectivity in etching with the appropriate acid/buffer combination has been used along with modal interactions and annealing to clearly differentiate two types of index change: one at the core/cladding interface and one within the core. ${ }^{28}$ The latter is more relevant to the fundamental mode and was shown to be thermally more stable. ${ }^{28}$ One may conclude, therefore, that it is not necessarily obvious that the chemical changes observed at the core/cladding interface of the type reported by Vengsarkar et al. ${ }^{26}$ have anything to do with density changes and, therefore, any major role in determining the birefringence in irradiated and hydrogen-loaded optical fiber.

On the other hand, the second dipole-based model has similar challenges. The results by Erdogan and Mizrahi, ${ }^{17}$ for hydrogen-loaded fibers, pursuing experimental "proof" of dipole orientation based solely on the observation that one laser writing polarization gives a larger birefringence than the other, are equally inconclusive. One serious drawback remains: significant induced birefringence is still observed for $p$-polarized light. We suggest an alternative possibilitynamely, that the $s$-polarized light is aligned with the radial stresses induced by fiber fabrication where preferential structural or dipole alignment of defects at the core-cladding interface are present. The evidence comes from the observed chemical selectivity of etching in hydrogen-loaded fibers at the core-cladding interface described above. Thus the role of radial dipoles in distinguishing absorption may play a role in enhancing anisotropy between the two states, which gives rise to birefringence. This contribution is expected to be substantially larger than the index change directly due to dipole formation since the drawing process produces a large number of defects $\left(>10^{22} \mathrm{~cm}^{3}\right)$ that are radially aligned at the corecladding interface. ${ }^{21}$

Anisotropy exists in the fiber system and while the index change from dipoles alone may be too small to explain birefringence, the sensitivity of stress fields is not and hence stress anisotropy, enhanced by preferentially radially aligned defects discriminating further the absorption differences between $s$ and $p$, can lead to larger index anisotropy. Given that hydrogen acts in a way to reduce stress potentially through $\mathrm{OH}$ formation (see earlier) an asymmetric level of hydrogen reaction across the fiber may be critical in creating anisotropic stress and index-the results of Vengsarkar et al., ${ }^{26}$ rather than support significant form anisotropy, confirm a large difference in reactions at the core/cladding interface and hence stress relief occurs on one side but not on the other. The result of Meyer et l. $^{31}$ showing erasure and growth of birefringence can likewise be similarly explained if the amount of light penetrating the core is different for each writing beam polarization.

Another fundamental problem with a simplistic dipole model based on defect excitation generally is the distribution of defect sites arising from the inhomogeneous and amor- 
phous glass network, where many defects may not be aligned with the polarization but will be of sufficiently low energy to be excited by the polarized light. Consequently, the statistical excitation of defects oriented with the laser polarization in an amorphous system cannot be readily compared to that observed in crystals. Some of the referenced work by Erdogan and Mizrahi, ${ }^{17}$ namely, that of Poirier et al., ${ }^{25}$ make an important and related point of the complexities of the dipole model with respect to index change. They observed, in nonhydrogen-loaded germanosilicate optical fiber, that the index change associated with oriented defects is only a small contribution to the total index change. This, they note, is consistent with changes in the deep UV, well below the bleached absorption bands of the defects associated with dipoles, accompany most of the index change. These deep UV changes are associated with structural changes. The contribution by bleaching of the dipole bands, while small, correlates with a negative component ${ }^{32}$ possibly as a result of preferential stress increases (rather than relief as they noted prior to correction of the stress model $^{25}$ ). Therefore, dipole orientation based on defects within the network is unlikely to be involved with the bulk of the measured birefringence as the index change contribution has been observed to be too small.

It is interesting to note that Poirier et al. ${ }^{25}$ used a high birefringent fiber in some of their experiments but did not comment substantially on the existing defect anisotropy and preferential dipole alignment along the fast and slow axes that is present as a result of fiber manufacture. Their results, however, do show a role but as noted the contribution to the index change appears to be small. They did not use polarized writing beams to establish a direct enhancement in this fiber. The issue was explored by Niay et $a .^{33}$ and consistent with our proposal that preferential radial alignment of absorption sites may enhance anisotropy still further.

UV irradiation leads to significant changes in stress fields not only radially but also axially in the case of a periodic structure. Direct experimental verification of these axial fields has been presented ${ }^{34,35}$ and measurements of reasonable resolution have shown that stress at the core/cladding interface increases with irradiation both uniformly and asymmetrically. ${ }^{36}$ Hence, the simplest explanation for the origin of the optical waveguide birefringence in nonhydrogen-loaded optical fibers becomes birefringence arising as a result of stress anisotropy. The UV attenuation across the core which affects particularly the former might lead to significant stress field anisotropy, both radially and axially in a grating, at the core-cladding interface and even across the core. Their inter-relationship as a result of Poisson's ratio will complicate but not change the degree of sensitivity of stress to laser field anisotropy of one sort or another. Annealing of these effects should therefore occur at relatively low temperatures-surprisingly, we were unable to find any reports studying the effect of annealing on UVinduced birefringence, measurements which can be difficult to undertake by conventional means.

As can be gathered, in the case of hydrogen-loaded optical fibers, which is of more commerical relevance, the situation is more complicated, in part by the continuing debate on what are the major contributions to UV-induced index change itselt let alone birefringence. Many previous theories have had to be discarded after experimental evidence has revealed that hydrogen-loaded fibers have two chemically and spatially distinct processes occurring at the core/cladding interface and within the core. ${ }^{28}$ The catalytic role of hydrogen, ${ }^{3-5}$ now widely accepted, ${ }^{37-39}$ is critical to this differentiation and has permitted a time scale separation of the two processes since one is driven by the other. ${ }^{7}$ Practical hypersensitization, itself a catalytic process dependent on hydrogen that extends the basic index change in nonhydrogenated optical fibers and is underpinned qualitatively by a two-step photosensitive mechanism, ${ }^{3-5}$ requires this time scale separation with the additional process at the core/ cladding interface so that it can in fact be avoided. Numerous benefits have stemmed from this but, from the time being, full hydrogen loading remains the primary method of photosensitization of optical fibers in commercial grating companies and we shall consider in this paper only fully hydrogenloaded optical fibers. However, future research on UVinduced birefringence in hypersensitized optical fibers, where it is likely to be smaller, will be of interest given their increased use in sensor and other advanced applications.

Therefore, the core/cladding interface of the fiber plays a role, including the suppression of stress through either the presence of molecular hydrogen and/or the formation of $\mathrm{OH}$ which itself appears to give rise to some structural dilation. Experimental evidence of the stress relaxation was observed by heating hypersensitized and fully hydrogen-loaded optical fibers - the latter, containing $\mathrm{OH}$, showed no elastic changes in fiber Bragg gratings whereas the former, where bulk of the hydrogen is out diffused before substantial $\mathrm{OH}$ can form, did. ${ }^{5}$ From arguments presented in the Introduction, the nature of the underlying causal factor that leads to index change in hydrogen-loaded optical fibers appears to be a contribution of both densification at the core through hydride formation, ${ }^{4}$ a small competing dilation, ${ }^{6,11}$ and a positive index change arising from polarizability changes as a result of all the chemical species generated, particularly $\mathrm{OH} .{ }^{11,40}$ It is clear that hydrogen in the core very early on leads to greater densification and hence larger index change than unloaded optical fibers and this factor dominates hypersensitized fibers. The degree to which all these contributions plays a role is openly debated and depends on a number of factors including fiber geometry, fiber preparation conditions including stress and quenching conditions, dopant concentration and distribution, and laser irradiation conditions. In the Introduction we noted that it has recently been proposed that the characteristic curve used to measure index change, either bulk or periodic, as a function of fluence or dose can provide useful insight to this and related problems. ${ }^{7}$ For example, in some cases hydrogen-loaded optical fiber, after a small initial nonlinear component, produces a very distinct linear response against fluence, ${ }^{41}$ which is indicative of a purely photorefractive change in index since the response time is local and fast and proportional to the formation of a hydrogen species such as $\mathrm{OH}$. On the other hand, densification involves structural relaxation in the glass and the generation of competing stresses. As is characteristic with any amorphous 
system, these processes will follow exponential-like decay where the exponent is a measure of the number and distributions and relaxations arising from a response to internal and external stresses and strains. The characteristic curve in these cases is usually expressed with index change as a function of the natural $\log$ of the fluence. Experimental evidence supporting this model include (1) UV annealing of stress in a planar waveguide leads to a linear index change versus natural $\log$ of fluence plot, ${ }^{42}$ indicating a single dominant bulk mechanical relaxation of the structure, (2) similar linearization of the characteristic curve of hypersensitized fiber, consistent with the removal of a second index process in the fiber leaving behind a dominant structural relaxation associated with densification, ${ }^{4,5}$ and (3) the linearization of the characteristic curve in radiation-exposed phosphosilicate fiber where electron generation and diffusion play the dominant $^{\text {role. }}{ }^{43}$

Hence, there is a substantive argument that the index change of fully hydrogen-loaded fibers has a contribution that may not be structural but is indeed photorefractive-in the proposal above, suggesting preferential radial alignment at the core-cladding interface is consistent with this. Likewise, the birefringence then arises from both chemical distribution anisotropy and stress anisotropy.

\section{MEASUREMENT OF BIREFRINGENCE}

\section{A. Precision phase-shifting gratings}

The measurement of birefringence using phase-shifted gratings has been previously reported. ${ }^{17,44-46}$ Phase-shifted gratings are gratings where two parts of the grating are offset from each other primarily in phase such that a notch in the transmission and reflection spectra (or windows in the grating stop gaps, depending on the language one feels more comfortable with) is generated. Since this notch is sensitive to the propagation constant of the traveling mode, when birefringence is present it will result in a split in its spectrum with two notch peaks separated by $\Delta \lambda$. The induced birefringence, $\Delta n_{x, y}$, where $x$ and $y$ denote the two orthogonal quasi-TE and TM eigenstates, is obtained from the wavelength splitting as ${ }^{47}$

$$
\frac{\Delta n_{x, y}}{n\left(\lambda_{B}\right)} \approx \frac{\Delta \lambda}{\lambda_{B}},
$$

where $n\left(\lambda_{B}\right)$ is the refractive index of the core at the Bragg wavelength $\lambda_{B}$. The resolution of this split within the notch is dependent on a number of properties, including where the phase shift is introduced within the grating and the magnitude of the phase, strength, and uniformity of the grating, which all affect the bandwidth of the notch. As well, the practical resolution of the available measurement technique will determine the actual resolution. When a high-resolution $(0.001 \mathrm{~nm})$ semiconductor tuneable laser source is used to measure the spectrum, it is extremely important to rule out laser drift, a common feature of high-resolution sources, particularly those that are wavelength swept. Once achieved, a resolution equal to the intrinsic birefringence is readily obtained-other methods using the direct laser line and a har- monic generated with an amplitude modulator allow $\Delta \lambda$ $<1 \times 10^{-10} \mathrm{~nm}$ to be resolved. ${ }^{45,46}$

Using a laser scanning source by any means restricts the use of simple adjustments of a polarizer during conventional birefringence measurements since the finite time between measurements makes laser drift and other factors impossible to separate, producing unreliable estimates of the birefringence, even when this birefringence is large. Such a method based on monitoring changes in the resonances in imperfect gratings which have the ends detuned from the center to create a Fabry-Perot notch filter was used by Erdogan and Mizrahi. ${ }^{17}$ However, splitting was not observed in a singlepass scanning mode and this approach is insufficient for any comprehensive comparison between different types of birefringence gratings. For this reason a high quality and reproducible method for producing phase shifts is essential.

Since the demonstration of a phase-shifted fiber grating for distributed feedback (DFB) and dense wavelength division multiplex (WDM) applications, ${ }^{48}$ by means of laser post processing, numerous methods have been developed, including using holographic phase masks with a $\Lambda / 4$ already present, ${ }^{49}$ the use of piezoelements to either overdither or physically displace a phase mask while scanning the UV writing beam, ${ }^{50}$ and the use of two polarizations in UV writing beam displaced from each other by a polarization beam splitter ${ }^{51}$ such that one half of the grating is one polarization out of phase with the other half, which is another polarization. This latter approach was based on the intrinsic advantages of the polarization control grating writing method. ${ }^{15,16}$ For our purposes the first two methods are unsatisfactory. Phase-shifted holographic masks do not always produce well-defined single-phase shifts but instead two-phase shifts, complicating the analysis of their spectra. ${ }^{52}$ Overdithering produces well-defined phase shifts but for unclear reasons, results in the literature show rather poor overall spectra compared with the expected spectra from simulation and an asymmetry that remains unaccounted for. ${ }^{53} \mathrm{dc}$ offsets using a piezo produce even worse results since the precision in displacement has to be significantly better than half of the fringe period desired ( $\ll 250 \mathrm{~nm}$ in physical displacement which is beyond the limits of many systems to produce reliably and reproducibility, even when combined with advanced interferometric monitoring despite piezo manufacturer and commercial grating supplier claims). Postprocessing remains the most popular choice of commercial manufacturers of phase-shifted devices such as fiber DFB lasers, ${ }^{54}$ since active monitoring allows precise adjustment without the interferometric precision that raises substantially the costs of gratings produced by alternative methods. Unfortunately, the additional complexity of understanding the impact on birefringence from a different fluence in the center compared to the rest of the grating ${ }^{48}$ makes this inappropriate for this work.

In this paper we have chosen to use a method where the precision in displacement is relaxed to a point where a $1-\mu \mathrm{m}$ displacement corresponds to a $<1-\mathrm{nm}$ step in fringe period. ${ }^{14}$ The precision phase-shifting method is based on a slightly tilted interferogram and, in this paper, we use it in combination with the polarization control grating writing method to produced three types of phase-shifted gratings: 


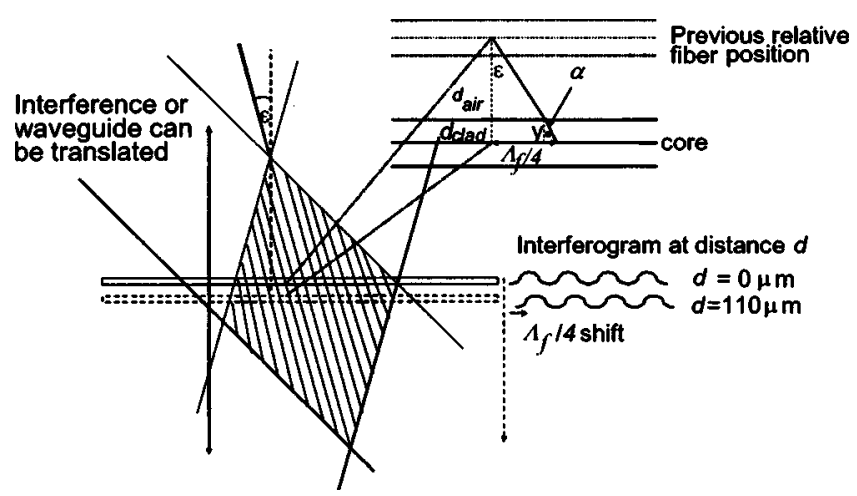

FIG. 1. Schematic of the precision phase-shifting (PPS) method we have recently reported (see Ref. 14).

one written with $s$-polarized light, the other with $p$-polarized light, and the third with the first half of the grating $s$ and the second half with $p$-polarized light.

The principle of the precision phase-shifting (PPS) technique is schematically illustrated in Fig. 1. By using the advanced polarization control grating writing method, ${ }^{15,16}$ schematically illustrated in Fig. 2, to implement PPS we can carry out the fabrication of all types of gratings with the one system without much effort. For an $s$ grating we use only $s$-polarized light and to introduce the desired phase shift we displace the fiber not along the direction of scanning but, instead, away from the phase mask. For a quarter wavelength displacement in optical fringe period the distance $d_{\text {air }}$ and a very small incidence angle for each polarization $\left(\varepsilon=0.17^{\circ}\right)$, the fiber must be translated away from the initial fiber position (or phase-mask surface) by

$$
d_{\mathrm{air}} \approx \frac{\Lambda_{f}}{4 \varepsilon}-\frac{r_{\mathrm{clad}}}{n\left(\lambda_{\mathrm{UV}}\right)} \approx \frac{\lambda_{\mathrm{Bragg}}}{8 \varepsilon n(\lambda)}-\frac{r_{\mathrm{clad}}}{n\left(\lambda_{\mathrm{UV}}\right)},
$$

where $\Lambda_{f}$ is the fringe period, $r_{\text {clad }}$ is the radius of the fiber cladding, and $n\left(\lambda_{\mathrm{UV}}\right)=1.5$ is the refractive index of silica at $248 \mathrm{~nm}$ (emission from a KrF laser). Experimentally we find the required distances to write close to $\pi$ phase shifted, $s$ and

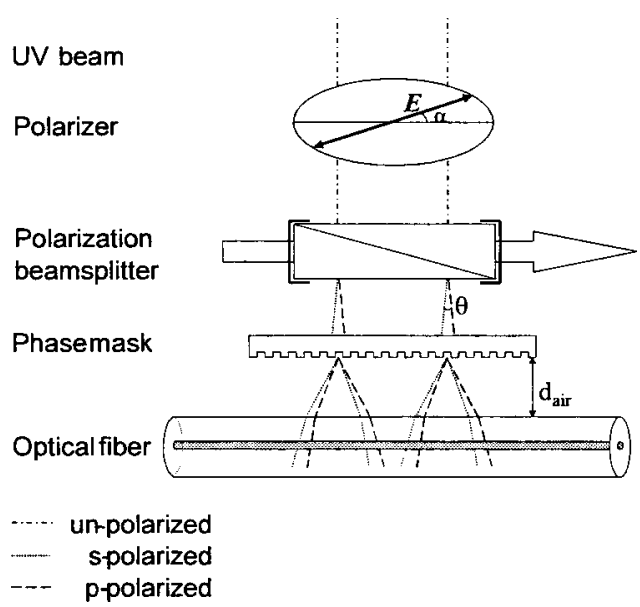

FIG. 2. Schematic of the arrangement of the polarization control method for grating writing (see Refs. 15 and 16). A polarizer allows selection of either $s$ or $p$ or both polarizations of the writing beam which strikes the optical phase mask at a small distance. The interferogram generated for individual polarizations is at a slight angle to the normal effectively simulating the PPS method. $p$ gratings at $248 \mathrm{~nm}$ are $(75 \pm 5) \mu \mathrm{m}$ and $(70 \pm 5) \mu \mathrm{m}$, respectively. Taking into account the cladding and index differences, this corresponds with a center core position from the original position of $\sim 110 \mu \mathrm{m}$. This is in good agreement with the figure calculated using the above expression. For the $p$-s grating the distance required to operate in the polarization control method where one half of the grating is $p$-polarized light and the other half is $s$-polarized light exactly $\Lambda_{f}$ displaced from each other is similarly calculated to be $68 \mu \mathrm{m}$ and experimentally verified to be $(70 \pm 5) \mu \mathrm{m} .{ }^{41}$

\section{B. Limitations of phase-mask-based grating writing systems}

The use of optical phase masks, even as simple beam splitters, can produce unforeseen complications. Some of the sources of error are obvious: the wavelength dependence of diffraction leads to a finite laser linewidth having a small angular wavelength spread that in most cases is only a problem in that the resulting degradation in grating quality is directly related to the coherence of the laser. These effects therefore tend to be negligible with most lasers. A much more significant problem, however, is beam divergence on phase masks. This leads to complex dephasing of the fringe profile along the mask length within the beam spot size leading to a partially self-apodized profile when divergence is low ( $\sim 0.5 \mathrm{mrad}$ for a spot size of a few millimeters). Because of the high sensitivity of the fringe period to displacement, noise can affect the quality of the grating and often unnecessarily complex feedback mechanisms to control noise in systems using phase masks, often with a degree of sophistication borrowed from gravitational wave interferometers, are sometimes employed. Nevertheless, since the phase shift is introduced physically in the center of the grating and apodization of the ends is not important, this latter issue need not be of particular concern in this work.

Finally, all these problems occur before other well known problems are considered-for example, the effects of even a few percent zero order ${ }^{55,56}$ and higher orders contributions can ruin the PPS technique when phase masks are used, and likely account for the observed "anomalous" results at given distances. ${ }^{14}$ Statistical errors in the phase-mask period and phase-mask tooth profile affect the quality further. Therefore, we undertook these experiments after careful consideration and taking care to ensure that the grating quality was sufficient for the purposes of this work, as Sec. V will demonstrate.

\section{UV-INDUCED BIREFRINGENCE RESULTS}

The fiber we have used in these experiments is a high concentration (typically $>20 \mathrm{~mol} \% \mathrm{GeO}_{2}$ ) optical fiber produced by OFS Denmark: OFS highly nonlinear fiber (HNFL); mode-field diameter $(\mathrm{MFD})=3.9 \mu \mathrm{m}$; cut-off $<1200 \mathrm{~nm}$ ). ${ }^{57}$ As such the fiber numerical aperture (NA) is higher than standard optical fiber and the core dimensions are comparably smaller $(\mathrm{MFD}=3.9 \mu \mathrm{m})$ to ensure singlemode operation at $1.5 \mu \mathrm{m}$. The highly germanium-doped core is surrounded by a depressed fluorine-doped inner cladding. Even though it is not especially designed for grating 
TABLE I. Summary of the cumulative fluencies used for each half of each of the fabricated ratings.

\begin{tabular}{ccc}
\hline \hline Grating & $\begin{array}{c}\text { Cumulative fluence } \\
\text { first-half grating } \\
\left(\mathrm{kJ} / \mathrm{cm}^{2}\right) \\
(L=5.5 \mathrm{~mm})\end{array}$ & $\begin{array}{c}\text { Cumulative fluence } \\
\text { second-half grating } \\
\left(\mathrm{kJ} / \mathrm{cm}^{2}\right) \\
(L=5.5 \mathrm{~mm})\end{array}$ \\
\hline$s$ & $1.63(s)$ & $1.63(s)$ \\
$p$ & $1.65(p)$ & $1.65(p)$ \\
$p-s$ & $1.65(p)$ & $1.63(s)$ \\
\hline \hline
\end{tabular}

writing, it has a good photosensitivity due to the highly germanium-doped core, and gratings over $12 \mathrm{~dB}$ without loading are readily inscribed at $248 \mathrm{~nm}$. This is comparable with more common photosensitive fibers that use less germanosilicate (typically $12 \mathrm{~mol} \%$ ) but have added boron. In these experiments we use deuterium loading at 100 atm to further enhance the photosensitivity. The growth profile of this fiber is such that after a relatively low fluence, a linear characteristic curve is obtained consistent with a photorefractive index change associated with $\mathrm{OH}$ formation. Hence, this fiber has a near-ideal response for grating growth after a small initial fluence.

We used a pulsed KrF laser to inscribe the various gratings. Consequently, there is some debate concerning the induced birefringence of pulsed versus cw lasers, particularly as some reports have indicated that the two sources give rise to differences in induced anisotropy. ${ }^{58}$ However, one of the authors pointed out that pulsed laser work had employed a phase mask while cw work had employed direct writing through a free space interferometer. The effect of a few percent zero order was to generate a higher-order grating superimposed on the Bragg grating to give rise to a small amount of side diffraction. ${ }^{59}$ Hence no direct evidence exists of a fundamental reason why pulsed and cw sources should give rise to different UV-induced birefringence under normal grating writing conditions. In fact, work currently under preparation indicates that similar magnitudes of UV-induced birefringence are possible. ${ }^{60}$ Therefore the conclusions in this paper apply equally to both pulsed and cw sources.

The gratings were produced as described in the earlier sections. A $\mathrm{KrF}$ unstable resonator source was used and the energy intensities of the laser at both polarization eigenstates were measured to be equal within $1.5 \%$ ( $p$ $=\sim 110 \mathrm{~mJ} / \mathrm{cm}^{2} /$ pulse; $s=\sim 109 \mathrm{~mJ} / \mathrm{cm}^{2} /$ pulse; repetition rate: $40 \mathrm{~Hz}$ ). All $11 \mathrm{~mm}$ long gratings were produced under similar conditions-Table I summarizes the fluencies for each grating. The Bragg wavelengths were kept within $1 \mathrm{~nm}$ of each other to ensure similar applied axial tensions.

Table II is a summary of the results obtained for the phase-shifted gratings. Also shown are figures measured for bulk-exposed samples at similar fluencies by conventional Jones matrix analysis. ${ }^{41}$ Once deuterium loading is complete (100 atm, 1 week), the transmission and reflection spectra were taken during and after grating writing using the setup shown in Fig. 3. Since the information provided by each propagation direction is, by reciprocity, identical, we concentrate on the transmission spectra in this paper. A wavelength swept tuneable laser source is monitored directly on a syn-
TABLE II. Summary of the measured birefringence results before and after annealing. For comparison we have added values obtained form growth curves of induced birefringence of bulk exposures measured by conventional means.

\begin{tabular}{ccc}
\hline \hline & Birefringence & $\begin{array}{c}\text { Birefringence } \\
\text { after annealing })\end{array}$ \\
\hline intrinsic & $(1.5 \pm 0.5) \times 10^{-6}$ & $(1.5 \pm 0.5) \times 10^{-6}$ \\
$p$ & $(3.0 \pm 0.5) \times 10^{-6}$ & $\left(\mathrm{H}_{2}\right.$ present $)$ \\
& $\mathrm{a}(2.0 \pm 0.5) \times 10^{-6}$ & $(1.5 \pm 0.5) \times 10^{-6}$ \\
$p-s$ & $(7.0 \pm 0.5) \times 10^{-6}$ & $\left(\mathrm{H}_{2}\right.$ present $)$ \\
$s$ & $\mathrm{a}(6.0 \pm 0.5) \times 10^{-6}$ & $(1.5 \pm 0.5) \times 10^{-6}$ \\
$\left(\mathrm{H}_{2}\right.$ present $)$ \\
$s$ & $(11.0 \pm 0.5) \times 10^{-6}$ & $(1.5 \pm 0.5) \times 10^{-6}$ \\
$\left(\mathrm{no} \mathrm{H}_{2}\right.$ present $)$ \\
$($ sample 2) & $(6.0 \pm 0.5) \times 10^{-6}$ & $(1.5 \pm 0.5) \times 10^{-6}$ \\
$s$ & $\mathrm{a}(4.0 \pm 0.5) \times 10^{-6}$ & $\mathrm{NA}$ \\
$($ standard $)$ & $\mathrm{b}(15.0 \pm 5) \times 10^{-6}$ & $\mathrm{NA}$ \\
$p$ & $\mathrm{~b}(1.0 \pm 0.5) \times 10^{-6}$ &
\end{tabular}

${ }^{\mathrm{a}}$ Measurements after initial relaxation over $24 \mathrm{~h}$ at $22^{\circ} \mathrm{C}$.

${ }^{\mathrm{b}}$ Figures obtained for the same fluence using the same laser from growth curves reported by Plougmann. ${ }^{41}$

chronized optical spectrum analyzer, allowing the spectral resolution to be determined by the laser step size $\sim 1 \mathrm{pm}$. A longer time scale drift of $3 \mathrm{pm}$ between scans, partly due to noise and temperature drift, does not interfere with the measurements since they are rapid and single pass. This contrasts with the standard measurement where adjustment of a polarization controller and measurement process has to be carried out for each polarization individually. ${ }^{60}$ Adjusting the two polarizers enabled launching of either single or both polarization eigenstates. By way of example, typical spectra showing the birefringence splitting in the phase-shift notch for the three types of gratings is shown in Fig. 4. Also shown are the reasonable fits obtained by transfer-matrix coupledmode theory simulations using the given experimental parameters. It is observed that $p-s$ gratings are the strongest, and $s$ gratings are the weakest, the difference larger than expected from the $1.5 \%$ difference in energies measured at the phase-mask surface. However, because the fringe contrast was determined by the position away from the phase mask, the exact contribution of zero-order interference is not the same for each grating, particularly between $p$ - $s$ and the other single polarization gratings since the latter involved a further displacement away from the mask during the inscrip-

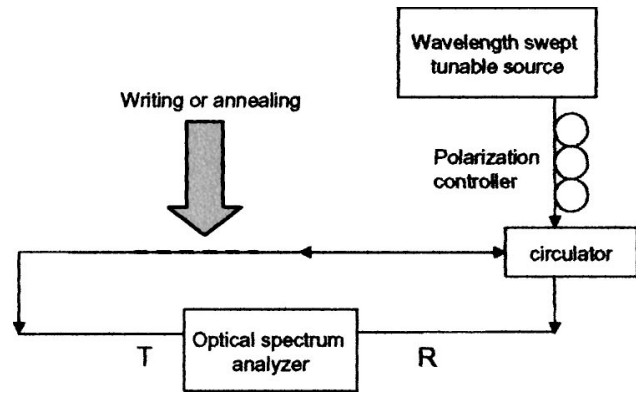

FIG. 3. Measurement setup for birefringence based on notch splitting in phase-shifted gratings. 


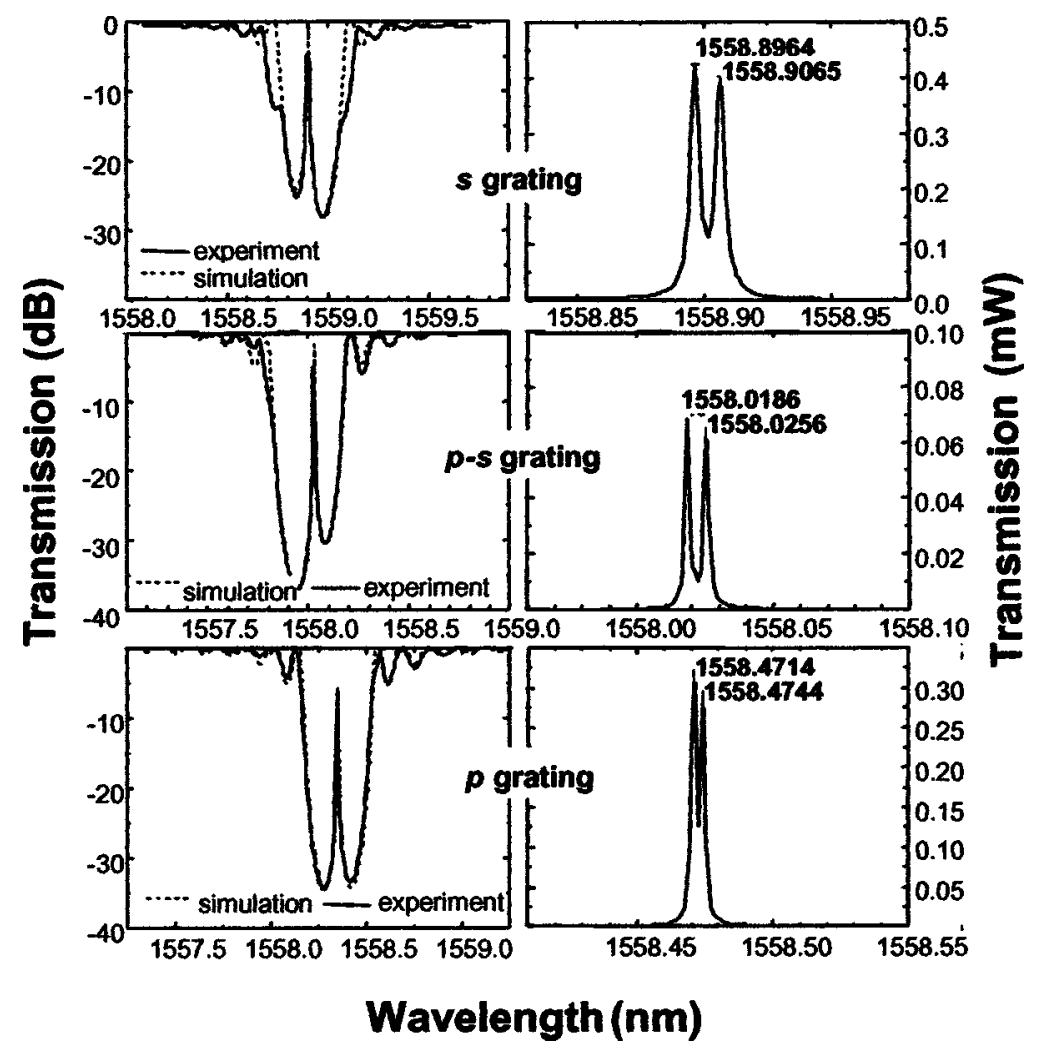

FIG. 4. Summary of transmission spectra obtained for the three types of phase-shifted grating and the corresponding notch spectra on the right (resolution: $1 \mathrm{pm}$ ). Splitting is clearly observed. The uniformity of the gratings is highlighted by the simulations which take into account differences in grating strength between $s$ and $p$ and the uncertainty in phase shift that can arise from additional contributions such as zero-order interference for the $p-s$ grating.

tion process. It was observed that $p$ gratings were always stronger than $s$ gratings and this difference within the $p-s$ grating could account for the observed deviation away from a $\pi$ phase shift. This difference may also suggest an attenuation difference between $p$ - and $s$-polarized writing light.

The notch splitting is best highlighted in linear transmission, as shown on the right of the figure. The splitting is readily observed using this method and the best visual resolution is obtained by operating in frequency mode on the optical spectrum analyzer. The grating written with entirely $s$-polarized light has the largest splitting and it was observed that this varied over successive grating writing between 9 and $10 \mathrm{pm}$ for the given fluence, which we attribute in part to an intrinsic form birefringence of 1-2 pm, a reasonable value given the high germanium concentration of the fiber. The gratings written with $p$-polarized light had the lowest splitting which averaged $\sim 3 \mathrm{pm}$. Both these values compare well with the bulk measurements for $s$ - and $p$-polarized light for the given fluence, indicating that, for birefringence at least, there is little influence on the birefringence from axial stress variations that affect the average index of a grating. Lying in between these two are the $p-s$ gratings with a splitting that averages $\sim 7 \mathrm{pm}$. All the gratings showed a small amount of relaxation over a period of one day, consistent with previous reports by Meyer et al. ${ }^{61}$ of similar relaxation for bulk exposure with $s$-polarized light.

Thus the method of measuring birefringence by splitting in the phase-shift notch is accurate and reliable, relaxing significantly the stringent requirements conventional means necessitate before the measurements can be accepted. Conversely, the phase-shifted method also affirms the reliability of recent results using bulk measurements. ${ }^{60}$ Further improvements in resolution are unnecessary since most fibers have a typical form birefringence of $\sim 1 \mathrm{pm}$. Within experimental uncertainty the birefringence measured for the bulk exposures by standard means are in agreement with those measured by phase-shifted grating. Hence, some justification for past correlations between bulk measurements and real periodic gratings when analyzing grating polarization mode dispersion (PMD) exists. The results also indicate that axial stresses do not play a major role in the induced birefringence. Since the role of axial stresses in a periodic grating are known to give rise to significant differences in induced index between bulk and periodic exposures in optical fibers, ${ }^{62}$ this is an important indication that the origins of birefringence and index change are not the same and that therefore geometric/form index anisotropy is not responsible for the birefringence.

\section{EFFECT OF ANNEALING AND HYDROGEN OUT DIFFUSION ON UV-INDUCED BIREFRINGENCE}

Absent from the literature is any report on annealing of UV-induced birefringence in optical fibers. Given that this is an ideal way to determine the general robustness of the induced birefringence, we carried out a simple annealing of the gratings with the induced birefringence. The experiments were set up a day after the grating writing and characterization had taken place and it was observed that even over this short time a noticeable decrease in splitting could be measured for all the gratings. Table II also highlights this difference. Consequently, two types of annealing data were recorded. 


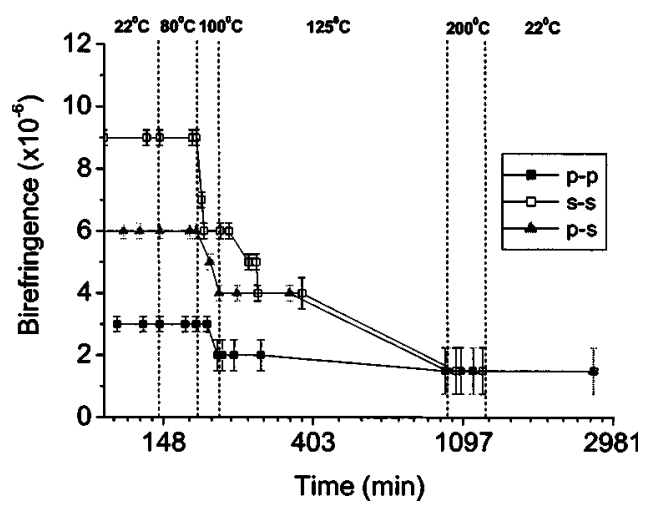

FIG. 5. Isochronal annealing of the birefringence in the various gratings when hydrogen is still present. The grating is eventually brought back to room temperature $\left(22^{\circ} \mathrm{C}\right)$.

\section{A. Isochronal annealing}

In these experiments, the gratings were ramped incrementally to various temperatures, up to $200{ }^{\circ} \mathrm{C}$, and then cooled down back to room temperature at $22^{\circ} \mathrm{C}$. Their spectra and splitting were monitored for a period of time at these set temperatures using the setup illustrated in Fig. 3. Figure 5 shows the results of this experiment. The resolution error is the laser step size which is $\sim 1 \mathrm{pm}$. However, when the splitting is below 2 pm it becomes increasingly difficult to determine the separation and consequently the error bars reflect the additional uncertainty at these levels. All the gratings experience annealing out of the notch splitting at 100 and $125^{\circ} \mathrm{C}$, an extremely encouraging result since it implies that the UV-induced birefringence is relatively unstable even at moderate temperatures and can be annealed out during the normal annealing stage used in, for example, commercial grating fabrication to stabilize index change. The birefringence was annealed out at $125^{\circ} \mathrm{C}$ over a short time period with no significant decrease in grating strength. Above $200{ }^{\circ} \mathrm{C}$ the grating began to anneal out the unstable component to index change, indicating further that the birefringence is not directly related to the index change. No recovery of the birefringence is observed at room temperature, suggesting the changes are permanent and not photoelastic. ${ }^{63}$

\section{B. Room-temperature hydrogen out diffusion and subsequent postannealing}

To explore the initial relaxation observed with the gratings, consistent with the work of Meyer et al., ${ }^{61}$ a second $s$ grating was fabricated and allowed to sit at room temperature. Since the initial decay shown in Table II was over one day, the possibility that the birefringence was related to the presence of molecular hydrogen was considered. Hence the decline in birefringence should correlate with hydrogen out diffusion. The hydrogen was allowed to out diffuse on the shelf over 16 days at room temperature $\left(22{ }^{\circ} \mathrm{C}\right)$. Figure 6 shows that apart from the initial relaxation, no decay of the birefringence was observed during the out-diffusion process. After out diffusion, annealing was conducted at $100{ }^{\circ} \mathrm{C}$ for half an hour. A small decrease is observed but no significant decay. The temperature was raised to $125^{\circ} \mathrm{C}$, leading to rapid annealing of the birefringence within resolution of the

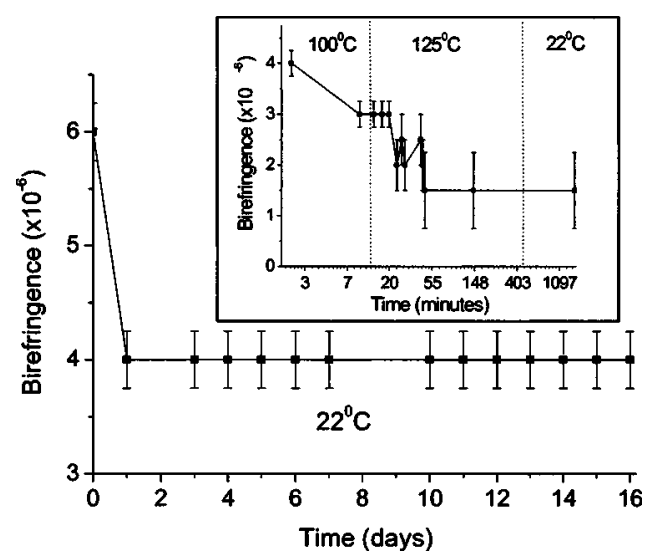

FIG. 6. Measured birefringence of an $s$ grating during hydrogen out diffusion at room temperature and (inset) annealing and cooling after complete out diffusion (resolution: $1 \mathrm{pm}$ ).

experiment, analogous to the previous results when hydrogen was present during annealing. No recovery of the birefringence was observed upon cooling to room temperature, ruling out photoelastic contributions. Hence we can conclude that molecular hydrogen, while involved in creating the birefringence, is not involved with annealing of the birefringence. An example of the notch profile observed when launching a $45^{\circ}$ polarized light into the $s$ grating after annealing is shown in Fig. 7.

\section{DISCUSSION OF RESULTS}

From all the results described thus far, we can infer that the process of inducing birefringence is more complex than has been previously appreciated. The initial drop in birefringence after writing and at room temperature over $24 \mathrm{~h}$ indicates that relaxation of the structure as the hydrogen begins to diffuse throughout the fiber as well as out of the fiber has occurred. Alternatively, some structural relaxation results in the randomization of any generated dipoles, although this seems unlikely since the $p$ grating shows this relaxation $(20 \%-30 \%)$ as well. A more plausible argument we propose is that since molecular hydrogen within the glass causes the network to swell (analogous to that observed in materials generally and described earlier), and thereby reducing existing core/cladding tensile stresses, subsequent depletion of hydrogen asymmetrically across the fiber as a result of an

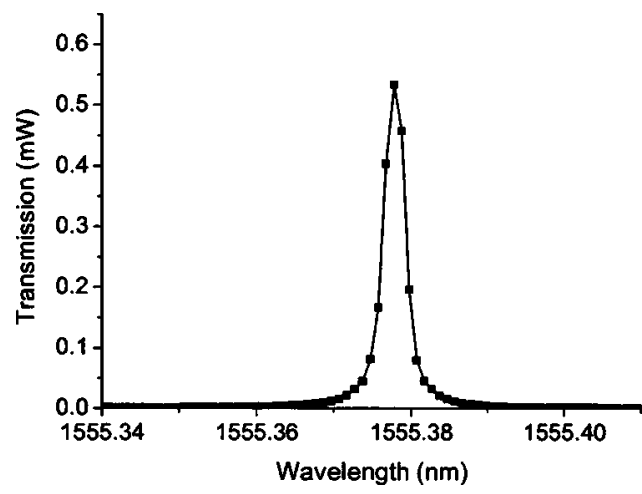

FIG. 7. Notch spectrum of an $s$ grating after annealing and cooling back to room temperature (resolution: $1 \mathrm{pm}$ ). 
asymmetric UV attenuation is responsible for this contribution to UV-induced birefringence. The value appears to be $\sim 2 \times 10^{-6}$ in the $s$ and $p-s$ polarized gratings and $<1$ $\times 10^{-6}$ in the $p$-polarized grating (resolution limited), which is at least equivalent or greater than the intrinsic birefringence. Initial diffusion of hydrogen across the concentration gradients established by the anisotropy may lead to stress relaxation. The results of Meyer et al. ${ }^{61}$ which are more detailed over the time period examined, are consistent with this explanation.

Since complete annealing occurs at relatively low temperatures, we can further infer that the remaining induced birefringence is not due to significant structural anisotropy of the type speculated by Vengsarkar et $a l .{ }^{26}$ but, instead, must be related to either stress or a somewhat low-energy dipole orientation. However, since the $p$ grating should not lead to any dipole selectivity or orientation in the first place, and it anneals in a similar manner as the $s$ grating despite the magnitude difference, it can be concluded that dipoles, although probably present, are not responsible for the bulk of the birefringence. This is consistent with the claims of Poirier et $a l .{ }^{25}$ that any direct dipole contribution to index anisotropy is likely to be too small. The observed difference in magnitude between $p$ and $s$ gratings is not large and can be explained (as suggested in Sec. II) on the basis of a difference in absorption across the core and core-cladding interfaces. The $s$ grating is nearly $5 \mathrm{~dB}$ weaker than the $p$ grating (Fig. 4), providing some evidence of this attenuation difference, either from scattering or absorption. Given the complexity of the fringe profile whenever using an optical phase mask, this general observation was repeated by writing further gratings. The ability to potentially distinguish this dependency further differentiates the phase-shift method with that of direct bulk measurements. We know from the initial relaxation observed at room temperature for all gratings that there is asymmetry in the induced changes and that this should correspond to the depleted molecular hydrogen which has since reacted in the glass. Since $\mathrm{OH}$ is implicated with dilation in a glass structure, the asymmetry is therefore likely to be related to the relaxation of stress in this region. Further, it is not unreasonable to suppose that an absorption difference between $s$-and $p$-polarized lights occurs because the $s$ state is closest aligned in the direction of the radial stress, where the defect orientation may already be preferentially aligned. This may account for all the large differences between $s$ and $p$ rather than scattering. Chemical selectivity is known to occur at the core/cladding boundary most probably due to the stresses that exist and it is this observation that Vengsarkar et al. ${ }^{26}$ actually make, so the role of stress is likely to be critical. Low-temperature heating then allows the fiber to accommodate this anisotropic change in stress by equilibrating as much as possible the overall stresses-effectively anisotropy relief.

The immediate benefits in the low stability of the birefringence are significant-the complex ways people have used to mitigate induced birefringence in DFB fiber lasers and PMD in dispersion compensating gratings, for example, can potentially be unnecessary provided the intrinsic birefringence can be removed. Another example is the use of pretwisting during DFB fiber inscription to eliminate the notch splitting and hence get laser action on one polarization only, ${ }^{64}$ which may not survive annealing itself. In principle, the concern of UV-induced birefringence raised by previous authors is allayed since the intrinsic birefringence can be calculated for with no need to accommodate a variable UVinduced birefringence in a grating device. The results also show that $p$-polarized light remains the most efficient way of optimizing the phase-mask interferogram within an optical fiber. Recent measurements show there is no significant difference in UV-induced birefringence using $\mathrm{cw}$ and pulsed lasers, ${ }^{60}$ further supporting a general mechanism where absorption differentiation is achieved between the two polarization states as a result of radial stresses introduced during fiber manufacture. Hence, there is no reason that the UVexciplex laser workhorse need perform any worse than any other UV source operating at similar wavelengths. This is becoming increasingly important for fiber materials that require wavelengths not readily accessible to solid-state lasers, such as $193 \mathrm{~nm}$. All these factors point to an improved overall understanding based on an appreciation of the complex contributions in glass waveguides and will lead to better quality gratings generally with lower noise and induced PMD.

\section{CONCLUSIONS}

In conclusion, we have demonstrated a precision phaseshifting approach to make various phase-shifted gratings using different combinations of polarized UV light. In doing so we confirmed the difference between $s$ - and $p$-polarized light reported by others and revealed the added complexity that hydrogen plays in the UV-induced process. We note that the birefringence is made up of at least two components with different thermal stabilities, one consistent simply with molecular hydrogen being present in the system. The birefringence, by deduction, appears associated with anisotropy in hydrogen reactions within the fiber. As a result they lead, through known mechanisms of dilation in glass, to anisotropic stress relaxation which can be annealed out, with or without hydrogen remaining, at low temperatures close to $125^{\circ} \mathrm{C}$. The annealing behavior is similar between the two polarization eigenstates, ruling out a significant dipole contribution. The anisotropy appears to be dependent strongly on the optical methods used to process the grating suggesting that it can be compensated for within the writing system if necessary. The final conclusion is that the concern of UVinduced birefringence appears less critical than previously thought since it will disappear during the normal annealing process involved with most grating manufacture.

\section{ACKNOWLEDGMENTS}

OFS Denmark is thanked for supplying the HNLF fiber. One of the authors (J.C.) acknowledges financial support from the Otto Mønsted Fond.

${ }^{1}$ P. J. Lemaire, R. M. Atkins, V. Mizrahi, and W. A. Reed, Electron. Lett. 29, 1191 (1993)

${ }^{2}$ J. Canning, Opt. Lett. 29, 815 (2004).

${ }^{3}$ J. Canning, R. Pasman, M. G. Sceats, and P. A. Krug, Proceedings of 
Conference on Photosensitivity and Quadratic Non-Linearity in Glass Waveguides, Portland, Oregon, USA, 1995 (Optical Society of America, Washington DC), p. 86

${ }^{4}$ J. Canning, Opt. Fiber Technol. 6, 275 (2000).

${ }^{5}$ J. Canning, Mater. Forum 25, 60 (2001).

${ }^{6}$ J. Canning and P.-F. Hu, Opt. Express 9, 476 (2001).

${ }^{7}$ J. Canning, J. Chem. Phys. 120, 9715 (2004).

${ }^{8}$ J. Canning, M. G. Sceats, H. G. Inglis, and P. Hill, Opt. Lett. 20, 2189 (1995).

${ }^{9}$ J. Canning, A. L. G. Carter, and M. G. Sceats, J. Lightwave Technol. 15 , 1348 (1997).

${ }^{10}$ C. K. Van Preski, R. Morton, and Z. Bor, J. Non-Cryst. Solids 265, 285 (2000).

${ }^{11}$ C. M. Smith, N. F. Borelli, J. J. Price, and D. C. Allen, Appl. Phys. Lett. 78, 2452 (2001)

${ }^{12}$ T. A. Ryumshina and S. P. Illyashenko, Int. J. Hydrogen Energy 24, 825 (1999).

${ }^{13} \mathrm{G}$. Alefeld and G. Volkl, Hydrogen in Metals (Mir, Moscow, 1981).

${ }^{14}$ J. Canning, H.-J. Deyerl, and M. Kristensen, Opt. Commun. 244, 187 (2004).

${ }^{15}$ J. B. Jensen, N. Plougmann, H.-J. Deyerl, P. Varming, J. Hubner, and M. Kristensen, Opt. Lett. 27, 1004 (2002).

${ }^{16}$ H.-J. Deyerl, N. Plougmann, J. B. Jensen, F. Floreani, H. R. Sørenson, and M. Kristensen, Appl. Opt. 43, 3513 (2004).

${ }^{17}$ T. Erdogan and V. Mizrahi, J. Opt. Soc. Am. B 11, 2100 (1994).

${ }^{18}$ J. Canning (unpublished).

${ }^{19}$ E. W. Taylor, V. R. Wilson, M. L. Vigil, R. A. Lemire, and E. E. Thompson, IEEE Photonics Technol. Lett. 1, 248 (1989).

${ }^{20}$ D. Wong, S. B. Poole, and M. G. Sceats, Opt. Lett. 17, 1773 (1992)

${ }^{21}$ M. G. Sceats, G. R. Atkins, and S. B. Poole, Annu. Rev. Mater. Sci. 23, 381 (1999).

${ }^{22}$ P. Y. Fonjallaz, H. G. Limberger, R. P. Salathe, F. Cochet, and B. Leuenberger, Opt. Lett. 20, 1346 (1995).

${ }^{23}$ M. G. Sceats and J. Canning, Proceedings of Summer School on Photosensitivity in Optical Waveguides and Glasses, Vitznau, Switzerland, 1998, edited by H. G. Limberger (IOA, Laussanne, Switzerland, 2000).

${ }^{24}$ J. Lauzon, D. Gagnon, S. LaRochelle, A. Blouin, and F. Ouellette, Opt. Lett. 17, 1664 (1992)

${ }^{25}$ M. Poirier, S. Thibault, J. Lauzon, and F. Ouellette, Opt. Lett. 18, 870 (1993).

${ }^{26}$ A. M. Vengsarkar, Q. Zhong, D. Iniss, W. A. Reed, P. J. Lemaire, and S G. Kosinski, Opt. Lett. 19, 1260 (1994).

${ }^{27}$ B. El-Kareh, Fundamentals of Semiconductor Processing Technologies (Kluwer Academic, Dordrecht, The Netherlands, 1995).

${ }^{28}$ J. Canning, K. Sommer, M. Englund, and S. Huntington, Adv. Mater. (Weinheim, Ger.) 13, 970 (2001).

${ }^{29}$ V. Mizrahi, P. J. Lemaire, T. Erdogan, W. A. Reed, D. J. DiGiovanni, and R. M. Atkins, Appl. Phys. Lett. 63, 1727 (1993).

${ }^{30}$ J. Canning and M. G. Sceats, Opt. Lett. 19, 119 (1994).

${ }^{31}$ T. Meyer, P. A. Nicati, D. Varelas, H. G. Limberger, and R. P. Salathe, Opt. Lett. 21, 1661 (1996).

${ }^{32}$ S. Bardal, A. Kamal, and P. St. J. Russell, Opt. Lett. 17, 411 (1992).

${ }^{33}$ P. Niay, P. Bernage, T. Taunay, M. Douay, E. Delavaque, S. Boj, and B. Poumellec, IEEE Photonics Technol. Lett. 7, 391 (1995).

${ }^{34}$ B. Poumellec, White Nights' Summer School on Photosensitivity in Optical Waveguides and Glasses, State Educational Centre, St. Petersburg, Russia, edited by H. Limberger (IOA, Laussanne, Switzerland, 2002).

${ }^{35}$ K. W. Raine, R. Feced, S. E. Kanellopoulos, and V. A. Handerek, Proceedings of 4th Optical Fibre Measurements Conference (OFMC '97) (National Physical Laboratory, Teddington, UK, 1997), pp. 200-203.
${ }^{36}$ H. G. Limberger, White Nights' Summer School on Photosensitivity in Optical Waveguides and Glasses, State Educational Centre, St. Petersburg, Russia, edited by H. Limberger (IOA, Laussanne, Switzerland, 2002).

${ }^{37}$ N. K. Viswanatham and J. F. Brennan, Proceedings of Conference on Optical Fibre Communication, Technical Digest Series, 2002 (Optical Society of America, Washington, D.C., 2002), Vol. 70, p. 107.

${ }^{38}$ B. Poumellec, P. Niay, M. Douay, and J. F. Bayon, J. Phys. D 29, 1842 (1996).

${ }^{39}$ M. Kristensen, Phys. Rev. B 64, 144201/1 (2001).

${ }^{40}$ M. Fokine, and W. Margulis, Opt. Lett. 25, 302 (2000).

${ }^{41}$ N. Plougmann, Ph.D. thesis, Technical University of Denmark, 2004.

${ }^{42}$ J. Canning, M. Åslund, A. Ankiewizc, M. Dainese, H. Fernando, J. K. Sahu, and L. Wosinski, Appl. Opt. 39, 4296 (2000).

${ }^{43}$ S. Girard, J. Keurinck, A. Boukenter, J.-P. Meunier, Y. Ouerdane, B. Azaýs, P. Charre, and M. Vie, Nucl. Instrum. Methods Phys. Res. B 215 , 187 (2004).

${ }^{44}$ J. L. Philipsen, M. O. Berendt, P. Varming, V. C. Lauridsen, J. H. Poblsen, J. Hubner, M. Kristensen, and B. Palsdottir, Electron. Lett. 31, 678 (1998).

${ }^{45}$ D. Yu. Stepanov, J. Canning, and Z. Brodzeli, NIST Technical Digest Symposium on Optical Fibre Measurements (OFM'98) (NIST, Bolder, CO, 1998), p. 149.

${ }^{46}$ J. Canning, D. Yu. Stepanov, B. Smith, Z. Brodzeli, and G. Yoffe, Proceedings of Summer School on Photosensitivity in Optical Waveguides and Glasses, Vitznau, Switzerland, 1998, edited by H. G. Limberger (IOA, Laussanne, Switzerland).

${ }^{47}$ J. L. Philipsen, M. O. Berendt, P. Varming, V. C. Lauridsen, J. H. Poblsen, J. Hubner, M. Kristensen, and B. Palsdottir, Electron. Lett. 31, 678 (1998).

${ }^{48}$ J. Canning and M. G. Sceats, Electron. Lett. 30, 1344 (1994).

${ }^{49}$ R. Kashyap, P. F. McKee, and D. Armes, Electron. Lett. 30, 1977 (1994).

${ }^{50}$ M. J. Cole, W. H. Loh, M. N. Zervas, and S. Barcelos, Electron. Lett. 31, 1488 (1995).

${ }^{51}$ K. P. Chuang, Y. Lai, and L.-G. Shen, IEEE Photonics Technol. Lett. 16, 834 (2004).

${ }^{52}$ Y. Sheng, J. E. Rothenberg, H. Li, Y. Wang, and J. Zweiback, IEEE Photonics Technol. Lett. 16, 1316 (2004).

${ }^{53}$ L. Poladian, B. Ashton, W. E. Padden, A. Michie, and C. Marra, Opt. Fiber Technol. 9, 173 (2003).

${ }^{54}$ M. Sejka, P. Varming, J. Hübner, M. Kristensen, Electron. Lett. 31, 1445 (1995).

${ }^{55}$ Z. Hegedus, Appl. Opt. 36, 247 (1997).

${ }^{56}$ J. Canning, M. G. Sceats, and S. Fleming, Opt. Commun. 171/4-6, 213 (1999).

${ }^{57}$ See www.ofs.dk for more information on the fiber used.

${ }^{58}$ M. Janos, J. Canning, and M. G. Sceats, Opt. Lett. 21, 1827 (1996).

${ }^{59}$ J. Canning, Proceedings of Optoelectronics and Communications Conference (OECC '2000), Makuhari Messe, Chiba, Japan, 2000 (Institute of Electronics, Information and Communications Engineers, Tokyo, Japan).

${ }^{60} \mathrm{H}$. R. Sørensen et al. (unpublished).

${ }^{61}$ T. Meyer, P. A. Nicati, P. A. Robert, D. Varelas, H. G. Limberger, and R. P. Salathe, Opt. Rev. 4, 53, (1997).

${ }^{62}$ D. Johlen, H. Renner, A. Ewald, and E. Brinkmeyer, Proceedings of European Conference on Optical Communications (ECOC'98), Madrid, Spain, 1998 (IEEE, NJ, 1998), p. 393.

${ }^{63}$ A. Hidayat, Q. Wang, P. Niay, M. Douay, B. Poumellec, F. Kherbouche, and I. Riant, Appl. Opt. 40, 2632 (2001).

${ }^{64}$ J. Canning and A. Michie, Australia, Patent No. PS2846, 7 June 2002 (2003). 